\title{
Heródoto y Argantonio: Un testimonio sobre la forma de poder en Tartessos
}

\author{
Álvaro Riaño Sanchez de la Poza *
}

\begin{abstract}
RESUMEN
El testimonio de Heródoto sobre el rey

Argantonio,y sobre la realidad sociopolítica del mundo tartésico puede

ser analizado desde el punto de vista

del vocabulario empleado por el historiador. Un estudio de los términos

con que Heródoto hace alusion a la estructura sociopolitica de Tartessos y a la naturaleza de su dirigente - palabras

como $\beta$ corizir o rupaveria-y y la comparación con otros ejemplos de su obra arrojarán alguna luz sobre esta cuestión. El análisis riguroso delas realidades internas de un texto histórico debe ser previo a su utilización como fuente, $y$ evita el riesgo de interpretaciones aventuradas.
\end{abstract}

PALABRAS CLAVE Heródoto, Argantonio. Tartessos, poder político, rey.

\section{SUMMARY}

Herodotus' testimony on king Argantonius as well as on the sociopolitical reality of the Tartessian world can be analysed according to the historian's usage of vocabulary. A survey on the terms Herodotus refers to the political structure of Tartessos. ant to its leader's features - words as

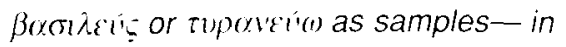
addition to the comparison to further examples of his work will indeed shed light to this topic. Therefore, an accurate analysis on the inner realities of a historical text will be previous to its use as a source and indoubtely it will avoid the risk of hazardous interpretations.

\section{KEY WORDS}

Herodotus, Argantonius, Tartessos. political power, king.

En el libro I de sus Historias. Heródoto introduce ciertas noticias acerca del enclave peninsular de Tartessos y de su longevo rey Argantonio, a propósito de las navegaciones de los foceos: 
"Estos foceos fueron los primeros griegos que hicieron largas travesías por mar, y fueron ellos los que descubrieron el Adriático. Tirrenia, Iberia y Tartessos. $Y$ navegaban, no en naves de carga, sino en naves de guerra de cincuenta remos. Una vez llegados a Tartessos se ganaron la amistad del rey de los tartesios, cuyo nombre era Argantonio, que ejerció el poder du rante ochenta años, y vivió en total ciento cincuenta" '.

Heródoto informa, además, que Argantonio invitó a los foceos a establecerse en el territorio tartésico, y que el rey les dio dinero en abundancia para que construyesen una muralla alrededor de su ciudad y pudiesen defenderse del avance de los persas en Asia Menor.

Estas referencias del historiador griego se vienen a unir al, por to demás, escaso repertorio de las fuentes literarias clásicas que nos han trasmitido algo sobre la existencia del mundo tartésico (G. Wagner, 1986a). Ahora bien, la escasez y dispersión de las noticias sobre Tartessos no ha impedido que, a lo largo de la historia de la investigación sobre la cultura tartésica, hayan surgido teorías muy elaboradas acerca de su estructura sociopolítica y de sus sistemas de poder. Estas teorías, contradictorias a veces, se han basado en interpretaciones también contradictorias de los relatos de los autores antiguos, o simplemente en dar más o menos crédito a éstos.

En una primera fase de las investigaciones sobre el mundo tartésico, una interpretación pretendidamente literal del relato de Heródoto marcó de manera singular el sentido de las elaboraciones teóricas de los estudiosos. La mención que el historiador griego hace de un "rey" ( $\beta \alpha \sigma i \lambda \varepsilon u ' s$ ) provocó que la investigación se centrase en la descripción de un reino con una estructura sociopolítica compleja, de un Estado basado en una organización socioeconómica plenamente urbana, y en la localización arqueológica de la ciudad capital de ese gran reino (Schulten, 1924; Antón, 1941; Bayerri, 1941).

El hecho es que la Arqueología ha sacado a la luz un horizonte cultural bien distinto al que, entre otros, Schulten, habian descrito como esperable en el ámbito tartésico. Ello ha conducido a que, en los últimos tiempos, los estudios sobre la estructura sociopolítica del mundo tartésico estén atravesando, en una comprensible reacción, por una fase hipercrítica con las fuentes literarias clásicas, por considerarlas, en su mayor parte, reelaboraciones de relatos legendarios, 0 , en todo caso, fundadas en referencias

Heródoto 1, 163. Todas las referencias al texto de Heródoto están tomadas y traducidas de la edición de Hude: Herodoti Historiae. Oxford, 1927. 
inseguras o lejanas. El razonamiento es sencillo: si informaciones como las que proporciona Heródoto han servido a historiadores modernos para postular erróneamente un tipo de sociedad urbana y estatal, hay que poner en entredicho ésta y otras fuentes antiguas. Asi, los términos con que Heródoto se refiere a la naturaleza del poder político en la cultura tartésica pasan por ser inadecuados o imprecisos, y se considera que el concepto de "realeza» $(\beta \alpha \sigma 1 \lambda \varepsilon i \alpha)$ es empleado "de forma indiscriminada para referirse a los sistemas políticos de los pueblos bárbaros» (G. Wagner, 1986a: 226).

Incluso historiadores que han venido postulando la existencia de navegaciones comerciales foceas en el litoral peninsular durante el siglo vi a. C., y que razonablemente podrían considerar fundado el conocimiento que en el medio griego se tenía de la realidad sociopolítica tartésica (y por tanto fiables las fuentes herodoteas), son reacios a dar una lectura plenamente histórica al pasaje de Heródoto, por creerlo excesivamente literario o legendario (Domínguez Monedero, 1996: 39).

En esta tendencia hipercrítica subyace, en realidad, una interpretación del texto de Heródoto muy similar a aquella por la que anteriores investigadores teorizaron sobre la existencia de un gran reino tartésico en el medio "orientalizante" de la Andalucía occidental. Se considera que Heródoto pretende testimoniar ese gran Estado territorial, gobernado por una realeza, y que por ello su testimonio es indigno de crédito. En definitiva, hasta ahora ha habido una única lectura del texto de Heródoto, al que se ha concedido un mayor o menor grado de veracidad o fundamento en función de las tendencias investigadoras.

Pues bien, creo que es necesario un análisis pormenorizado de los términos con que Heródoto se refiere a la naturaleza del poder político en Tartessos, personalizado en Argantonio, análisis que no debe quedar restringido al pasaje en cuestión, sino ser puesto en comparación con otros ejemplos de la obra de Heródoto. De esta manera será posible comprender el significado preciso con que el historiador de Halicarnaso emplea estos términos, y llegar a conclusiones ciertas sobre lo que quería expresar.

Pretendo, a través de este trabajo, dar una nueva perspectiva a la lectura de una fuente clásica (y quizá a la lectura de fuentes antiguas en general), que no merece ser desechada o hipervalorada en función de que nuestra interpretación de elia coincida en mayor o menor medida con una teoría previamente construida. La lectura debería ser lo más pormenorizada posible y la información, interdependiente pero no excesivamente supeditada al resto de los datos que aporta la investigación histórica. 
En el pasaje anteriormente citado, Heródoto afirma que los foceos "se ganaron la amistad del rey $(\beta \alpha \sigma \mid \lambda \varepsilon$ \&í) de los tartesios, cuyo nombre era

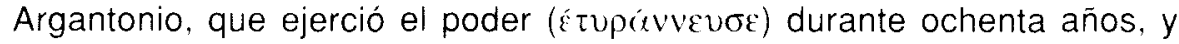
vivió en total ciento cincuenta".

Aparte del dato de la considerable longevidad de Argantonio, que se ha explicado por la existencia de una sucesión de varios reyes, ha causado sensible desconcierto entre los lectores de Heródoto el vocabulario con que el historiador se refiere al poder de Argantonio en Tartessos. Y así, tan pronto se ha considerado la existencia de una realeza tartésica equiparable a las grandes monarquias orientales como se ha afirmado que "para el padre de la historia, Argantonio fue un tirano del tipo de los de la Grecia Arcaica" (Blázquez, 1993: 134). Como hemos visto, Heródoto da a Argantonio el nombre de $\beta \alpha \sigma 1 \lambda \varepsilon[1$, “rey», y se refiere a su forma de ejercer el poder con el verbo tupovveú(1), "gobernar como tirano", en su interpretación más clásica. Ahora bien, esta combinación de palabras aparece con cierta frecuencia en la obra del historiador griego, y en cada una de estas ocasiones Heródoto se refiere de manera muy precisa a las circunstancias en que una o varias personas encabezan un sistema de poder. Un repaso del empleo de estos términos puede darnos la clave sobre su significado concreto.

El sustantivo $\beta(x \sigma i \lambda \varepsilon i s$ (y otras palabras relacionadas con esta raiz, como $\beta \alpha \sigma \mid \lambda \varepsilon u(1)$, "reinar», o $\beta\left(\alpha \sigma \nu \lambda \eta \eta^{\prime \prime \eta}\right.$, "realeza"), se emplea siempre para determinar la circunstancia en que una persona, por una suerte de legitimidad dinástica, o por su pertenencia a un grupo de parentesco capacitado por la razón que sea para dominar sobre otros, adquiere el derecho a ejercer un determinado poder. Es un error suponer que estos términos impliquen la existencia de una monarquia consolidada en una sociedad compleja. $\beta \alpha \sigma i \lambda \varepsilon r$ s, tal como aparece, por ejemplo, en Homero (Odisea 1, 394), es el jefe de un grupo de parentesco, en el marco de una sociedad gentilicia.

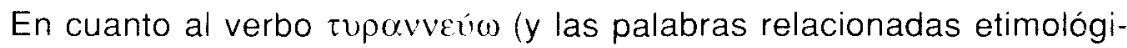

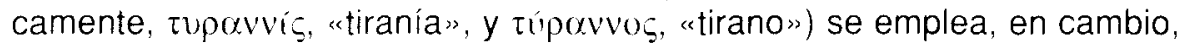
para referirse a la situación en que se ejerce el poder de una manera absoluta o despótica, independientemente de si a ese poder se ha accedido de un modo legítimo o ilegítimo.

De esta manera, los dos grupos de términos se utilizan juntos en numerosos pasajes de la obra de Heródoto, en los que es posible delimitar su sentido concreto.

Por ejemplo, es normal que aparezcan cuando Heródoto escribe sobre la historia del reino de Lidia: 
"... mencionaré a Ardis, hijo de Giges, que reinó ( $\beta \alpha \sigma i \lambda \varepsilon i \sigma_{0}\left(x^{\prime} \mathrm{To}_{\zeta}\right)$ después de Giges. Este conquistó Priene y se lanzó sobre Mileto. Y estando él

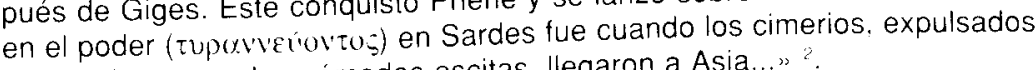
de sus tierras por los nómadas escitas, llegaron a Asia..."?

Parece claro que el verbo $\beta \alpha \sigma 1 \lambda \varepsilon v$ u( ) es usado por Heródoto en un contexto en el que desea explicar la legitimidad que ha llevado a Ardis al trono: es hijo de Giges y sucesor suyo. En cambio, el verbo tupavveú() indica aquí simplemente la propia ejecución de poder.

En el siguiente ejemplo, en que aparece el lidio Candaules, se muestra cómo no resultan incompatibles, por las razones que he expuesto, los sustantivos túpovvos y $\beta \alpha \sigma i \lambda \varepsilon u ́ s$ referidos a un mismo personaje, y que $\beta \alpha \sigma i \lambda \varepsilon u s$ en concreto se emplea preferentemente en aquellos contextos en los que se da cuenta de una sucesión dinástica o de los mecanismos sucesorios:

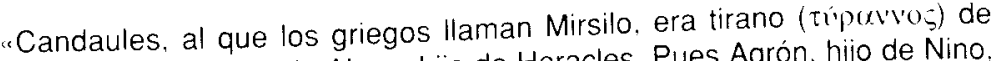
Sardes, y descendiente de Alceo, hijo de Heracles. Pues Agrón. hijo de Nino. hijo de Belo, hijo de Alceo, se convirtió en el primero de los descendientes de Heracles que fue rey $\left(\beta(\alpha) i \lambda \varepsilon i^{\prime}\right)$ de Sardes, y Candaules, hijo de Mirso, en el último $\approx$.

Se observa que el sustantivo típovvos aparece en un contexto en que no tiene trascendencia la legitimidad dinástica: los antepasados de Candaules, Alceo y Heracles, no fueron reyes de Sardes, y por tanto no se les nombra para explicar el poder del primero. Pero cuando Heródoto establece los términos de la dinastía heráclida en Sardes, emplea ya el sustantivo $\beta \alpha \sigma i \lambda \varepsilon$ í

Encontramos también la misma combinación de vocabulario en el libro dedicado a Egipto:

“Una vez libres los egipcios del reinado $(\beta \alpha \sigma i \hat{\lambda} \varepsilon \dot{ }(\alpha) \tau(x)$ del sacerdote de Hefesto, puesto que no eran capaces de estar largo tiempo sin rey ( $\beta\left(x u 1 \lambda \bar{\varepsilon}_{5}\right)$, dividiendo Egipto en doce partes, establecieron doce reyes $\left(\beta(x) 1 \lambda \varepsilon\left(\alpha_{5}\right)\right.$. Estos, concertando alianzas matrimoniales, comenzaron a reinar $\left(\hat{\varepsilon} \beta\left(x_{0} \lambda \hat{\varepsilon} v 0\right)\right.$ ) con el compromiso de no destronarse... $Y$ la razón de que establecieran estos pactos y de que los respetaran firmemente es

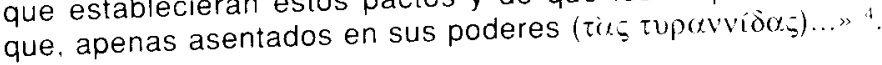

Heródoto 1, 15.

Heródoto 1.7.

Heródoto 2. 147 
Este pasaje es interesante por cuanto Heródoto parece recoger en él el significado más primitivo de $\beta \alpha \sigma 1 \lambda \varepsilon u ́ s$, es decir, el de jefe de un grupo de parentesco, que se relaciona con sus iguales por medio de alianzas matrimoniales, al modo en que ya he dicho que Homero emplea este sustantivo para designar a los caudillos aqueos.

También es frecuente esta combinación en los pasajes en que aparecen los reyes de los persas:

«Después de que realizó este ordenamiento y se afianzó en el poder (tî́ tup(xvidi) fue riguroso en la administración de justicia... Deyoces tuvo un hijo, Faortes, que al morir Deyoces, tras reinar $\left(\beta \alpha \sigma i \hat{n} \varepsilon i \sigma \alpha \nu^{\prime} \sigma_{\zeta}\right)$ cincuenta y tres años..." $\stackrel{5}{5}$.

Nuevamente se observa el empleo del verbo $\beta \alpha \sigma i \lambda \varepsilon u ́ s$ cuando se expone el parentesco por el que una persona accede a un trono o posición principal de un sistema de poder. En cuanto al sustantivo tupovvís, parece claro que se refiere a la propia ejecución del poder o a las medidas para ejercerlo. Por otra parte, es destacable que el sustantivo tupovvis tiene frecuentemente un sentido abstracto ( poder»), como aparece en el pasaje anterior y también en esta exhortación de Jerjes a Artabano:

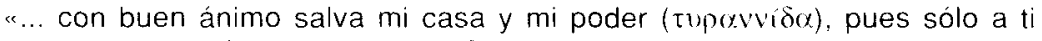
entre todos confio yo mis cetros" "

Para un griego, en todo caso, la forma de ejercer el poder que se expresa con el verbo tupavev́w o con el sustantivo túpavvos es independiente de si quien lo ejerce es o no un $\beta \alpha \sigma i \lambda \varepsilon u$ s. Por ello hay que insistir en que Heródoto emplea estas palabras con criterios muy precisos, y no arbitrariamente Por ejemplo, de un sátrapa nunca se dirá que es un $\beta \alpha \sigma i \lambda \varepsilon u ́ s$ ni nada similar, pues es claro que su posición es dependiente del Gran Rey:

"Estaba en el poder (ćtupávifue) en esa provincia Artayctes, un gobernador ( $i \pi\left(x_{\rho} \times \varphi_{\varsigma}\right)$ de Jerjes..." ".

Es el mismo caso que en los ejemplos siguientes:

«Eran Pigres y Mastyes dos peonios que, cuando Darío pasó a Asia,

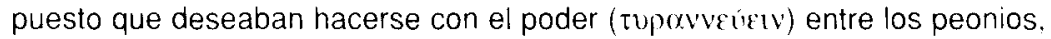

Heródoto 1. 100-102

Heródoto 7, 52.

Heródoto 9, 116. 
se presentaron en Sardes llevando consigo a una hermana suya, hermosa y de gran estatura..." $"$

"(Artafrenes) nombró general en jefe al persa Megabates, de la casa de los Aqueménidas, primo suyo y de Dario, y del que una hija, si es cierta la historia, casó tiempo después con el lacedemonio Pausanias. que quería convertirse en tirano ( $\tau$ i $p \alpha$ vos $)$ de Grecia" ?.

Aparte de estos ejemplos, tenemos todos aquéllos en que la raíz de Típavvos aparece referida a la historia de las ciudades griegas. En ellos, lo que interesa es definir una determinada forma de ejercer el poder por parte de los tiranos, pero al no existir rastro de esa especie de legitimidad dinástica de que ya he hablado, no se usa el término $\beta \alpha \sigma i \lambda \varepsilon \omega^{\prime}(1)$.

A la luz de los datos obtenidos en este estudio de vocabulario, creo posible extraer algunas conclusiones, que pueden permitir, en cierta medida, un redescubrimiento de Heródoto como fuente histórica en la cuestión de la estructura sociopolítica del mundo tartésico.

En primer lugar, no parece aventurado afirmar que Heródoto emplea los términos referidos a la realidad política de un modo consecuente y preciso, y no arbitrariamente. De los pasajes citados puede deducirse de manera bastante exacta el sentido con que estas palabras son usadas por el historiador, por lo que el texto en que se refiere a Argantonio parece describir una situación muy concreta en Tartessos: aquélla en que existe una forma de concentración de poder, ejercido de forma absoluta, poder al que una persona accede por la legitimidad que le confiere su pertenencia a un determinado grupo de parentesco, que posiblemente ha establecido un sistema de sucesión basado en este parentesco.

Parece difícil tratar de determinar el origen o alcance del poder ejercido en el mundo tartésico por dirigentes como Argantonio, utilizando exclusivamente esta fuente literaria. Lo cierto es que, a tenor de lo transmitido por Heródoto, tan inadecuado sería equiparar Tartessos con las grandes monarquías orientales como negar que pueda darse en esta zona peninsular un sistema político que implique una concentración hereditaria del poder.

Para que pueda darse esta concentración de poder, basta quizá suponer que determinados grupos eran capaces de controlar el acceso a los recursos mineros que parecen estar en la base de la riqueza de Tartessos. y de controlar además la mano de obra necesaria para explotarlos ( $G$. 
Wagner, 1992: 16-19; 1993: 109). Para postular esta capacidad de control no es necesario suponer la existencia de un sistema sociopolítico de tipo estatal con una base territorial.

El hecho de que los mecanismos de relación que proporciona el parentesco sean los más adecuados para que se produzca una incipiente jerarquización social, en el marco de una economía de redistribución de bienes y un sistema político de jefaturas (G. Wagner, 1992: 18-19) es coincidente en gran medida con el significado que, como ya he apuntado, tiene primariamente en griego el término $\beta \alpha \sigma i \lambda \varepsilon u \varsigma_{5}$, cuya traducción más clásica en castellano, "rey», es quizá la causa de muchos equivocos sobre su sentido en cada caso. Lo cierto es que sería necesario emplear en su traducción una más amplia gama de sustantivos en nuestra lengua, para poder dar cuenta de toda la serie de realidades sociopolíticas que esconde.

Un segundo aspecto de este tema del que me parece posible extraer conclusiones es el de la presencia griega en el mundo tartésico. En efecto, parece pertinente establecer una relación entre esta presencia y la cuestión de si Heródoto está en disposición, en función de la fiabilidad de sus propias fuentes, de ofrecer un testimonio fidedigno sobre la situación politica de Tartessos.

La discusión sobre las relaciones exteriores del mundo tartésico viene apoyándose en posiciones muy dispares, y la dificultad de reconocer que los griegos llegaron a tener, desde la primera mitad del siglo vi a.C. (que es cuando parece más probable que habría que situar el encuentro de los foceos con Argantonio), un razonable conocimiento del sudoeste peninsular estriba en suponer que el comercio fenicio en la zona debía ser monopolístico. Lo que parece más difícil de postular, en todo caso, es que en el ámbito tartésico pudiese existir un comercio griego de tipo empórico (Olmos Romera, 1989).

Entonces, ¿es posible calibrar, a través del texto de Heródoto, si el conocimiento que los griegos poseían del mundo tartésico era de primera mano o no?

Por una parte, la precisión terminológica que, según lo que he comentado más arriba, puede detectarse en los pasajes de Heródoto no parece adecuada a la reelaboración de un relato más o menos legendario. Es más lógico pensar que el historiador de Halicarnaso tenía entre manos una información que consideraba fidedigna. Aunque algunos aspectos puedan estar exagerados (como el ya citado de la longevidad de Argantonio), ni la distancia cronológica entre el historiador y la época supuesta de los acontecimientos ni el conocimiento geográfico que ya en tiempos de 
Heródoto debía tenerse de la Península predisponen a una mitificación de los hechos.

Por otra parte, las investigaciones arqueológicas en la ciudad de Huelva han sacado a la luz, para la primera mitad del siglo vi a.C., un notable incremento de cerámica griega, en relación a épocas anteriores (Cabrera Bonet, 1988: 53). El porcentaje de los hallazgos de cerámica griega sigue siendo insignificante en relación a los descubrimientos de productos fenicios, pero el dato de su incremento permite quizá replantearse la cuestión de la relación entre griegos y fenicios en el sudoeste peninsular (Fernández Jurado, 1986). Es dificultoso explicar cómo pudieron darse contactos entre foceos y tartesios en un ámbito amplia e interesadamente explotado por los fenicios. Pero si esos contactos, todo lo ocasionales que se quiera, existieron, habría que revalorizar el conocimiento griego de Tartessos, y considerar que Heródoto pudo tener acceso a fuentes de información razonablemente fundadas.

Las fuentes antiguas no deberian ser susceptibles de una interpretación interesada en función de modelos previamente concebidos, ni simplemente desechadas desde posiciones excesivamente críticas, pues ambas concepciones nacen frecuentemente de consideraciones apriorísticas que lastran de forma negativa el estudio del documento. Es cierto que, cuando se trata de establecer las relaciones entre la realidad y las fuentes, lo que éstas transmiten es una imagen mediatizada de aquella (Plácido, 1993: 81), pero ello no debe ser un pretexto para interpretar los testimonios en función de la mayor o menor utilidad que puedan suponer para el historiador. Un estudio riguroso y profundo de las implicaciones internas de los textos debe ser compatible con la información que proporcionan otras fuentes históricas.

\section{BIBLIOGRAFIA}

ANTON, F. (1941): "La ciudad de Tartessos-Tarxix. La isla de Saltes en Huelva y el imperio Ibero-Turdetano". Boletin de la Real Sociedad Geográfica. LXXV1l. págs. 443-484.

BAYFRRI, E. (1941): "En busca de la resolución del problema Tharsis-Tartessos". Boletin de la Real Sociedad Geográfica. LXXVII, págs. 736-758.

BLAzQUEZ. J. M. (1993): "El enigma de la religión tartésica". Los enigmas de Tarteso. Madrid. págs $117-136$

Cabrera Bonet. P. (1988): "El comercio foceo en Huelva:cronologia y fisonomía". Huelva Arqueológica. 10-11 (vol. 3), págs. 43-100.

Dominguez Monede ro. A. (1996): Los griegos en la Peninsula lbérica. Madrid.

FERNANDEl Jurado. J. (1986): «Fenicios y griegos en Huelva». Homenaje a Luis Siret. págs $562-574$

Gonzalt z. WAGneh. C. (1986a): "Tartessos y las tradiciones literarias". Rivista di Studi Fenici. 14 (2), págs. 201-218.

Gonzalez WAGNEF, C. (1986b): "Notas en torno a la aculturacion en Tartessos". Gerión. 4. págs. 129-160. 
Gonzalez Wagner, C. (1992): "La Historia Antigua y la Antropologia: el caso de Tartessos", Kolaios, 1 , págs $1-37$.

GonzALEZ WAGNER, C. (1993): "Las estructuras del mundo tartésico", Los enigmas de Tarteso. Madrid, págs. 103-113.

Olmos Romera, R. (1989): "Los griegos en Tarteso: una nueva contrastación entre las fuentes arqueológicas y literarias». Tartessos: arqueologia protohistorica del Bajo Guadalquivir. Sabadell, págs. 495-518.

PlACIDO, D. (1993): "La imagen griega de Tarteso", Los enigmas de Tarteso. Madrid, págs. 81 89.

Roull LaRD, P. (1991): Les Grecs et la Péninsule Iberique du vil au vi siècle avant Jésus-Christ. Paris.

SCHULTEN, A. (1924): Tartessos, Madrid (edición 1972) 